\title{
The Steiner Ratio Conjecture for Cocircular Points
}

\author{
J. H. Rubinstein ${ }^{1,2}$ and D. A. Thomas ${ }^{2}$ \\ ${ }^{1}$ Institute for Advanced Study, Princeton, NJ 08540, USA \\ ${ }^{2}$ Department of Mathematics, University of Melbourne, \\ Parkville, Victoria 3052, Australia
}

\begin{abstract}
A Steiner minimal tree $S$ is a network of shortest possible length connecting a set of $n$ points in the plane. Let $T$ be a shortest tree connecting the $n$ points but with vertices only at these points. $T$ is called a minimal spanning tree. The Steiner ratio conjecture is that the length of $S$ divided by the length of $T$ is at least $\sqrt{3 / 2}$. In this paper we use a variational approach to show that if the $n$ points lie on a circle, then the Steiner ratio conjecture holds.
\end{abstract}

\section{Introduction}

Let $X$ denote a set of $n$ points $\left\{x_{1}, x_{2}, \ldots, x_{n}\right\}$ in the plane $\mathbb{R}^{2}$. A Steiner minimal tree $S$ is a network of shortest possible length connecting these points. $S$ may have extra vertices called Steiner points. Let $T$ be a shortest tree connecting up the points of $X$ but with vertices only at the points $x_{1}, x_{2}, \ldots, x_{n}$. T is called a minimal spanning tree. Let $L_{S}, L_{T}$ denote the lengths of $S, T$, respectively, and let $\rho=L_{S} / L_{T}$. $\rho$ is called the Steiner ratio. Gilbert and Pollak [4] conjectured that $\rho \geq \sqrt{3} / 2$ and this has been shown to be true for $n=3[4], 4[5],[3], 5[2]$, and $6[7]$.

In this paper we show that if the $n$ points lie on a circle, the Steiner ratio conjecture holds. We use the variational approach discussed in detail in [6] and applied in [7]. In Section 1 we give a brief discussion of the variational technique and pose the Steiner ratio conjecture as a problem of variations. In Section 2 we give a proof of the Steiner ratio conjecture for $n$ cocircular points.

It suffices to assume the circle has unit radius and this convention holds throughout. 


\section{The Calculus of Variations and the Steiner Ratio}

To solve the Steiner ratio conjecture it suffices to look at the case when $S$ is a full Steiner tree, that is, has $2 n-2$ vertices. If $S$ is not full we can dissect $S$ into smaller full components as in [4]. Note that in each component the $x_{i}$ remain cocircular. As in [4], if the ratio conjecture holds in these smaller full components, it will hold for the given $X$.

Definition. The configuration space $\Delta_{0}$ is the collection of all sets of $n$ points $\left\{x_{1}, x_{2}, \ldots, x_{n}\right\}$ arranged on the unit circle.

Remarks. (1) For convenience here we allow the possibility that some of the points may coincide. Later we will need to suppose the points have a given cyclic order round the circle.

(2) Clearly, $\Delta_{0}$ is a product of $n$ circles, i.e., is the $n$-torus. In particular, $\Delta_{0}$ is compact.

Our strategy is to study the ratio function $\rho$ on $\Delta_{0}$. In particular, it is immediate that $\rho$ is continuous and hence attains a minimum at some configuration $Y$ in $\Delta_{0}$. If $\rho<\sqrt{3} / 2$ somewhere in $\Delta_{0}$, then certainly $\rho<\sqrt{3} / 2$ at $Y$. We show this is impossible, i.e, $Y$ cannot be a minimum at which $\rho<\sqrt{3} / 2$. This is established by studying the differential $D \rho$.

In [6] the differentiability of $\rho$ is discussed on the simplex $\Delta$ parametrized by the lengths of the edges of $S . \Delta_{0}$ is a subspace of the cone on $\Delta$ and so the observations of [6] apply to $\rho$ restricted to $\Delta_{0}$. Recall that the cone on $\Delta$ is obtained by relaxing the requirement that the sum of the lengths of the edges of $S$ be one.

Definition. A critical point for $\rho$ on $\Delta_{0}$ is a configuration $Y$ in $\Delta_{0}$ for which $D \rho(v) \geq 0$ for any vector $v$ at $Y$.

Note that $v$ is a tangent vector to $\Delta_{0}$. It can be shown by the formula for differentiating quotients $[6$, Lemma 1$]$ that

$$
D \rho(v)=\left(\dot{L}_{T} / L_{T}\right)\left(\dot{L}_{S} / \dot{L}_{T}-\rho\right),
$$

where $\dot{L}_{T}=D L_{T}(v), \dot{L}_{S}=D L_{S}(v)$. As a corollary $[6$, Corollary 1$]$, if $\dot{L}_{T}<0$ $\left(\dot{L}_{T}>0\right)$, then $D \rho(v) \geq 0$ is equivalent to $\dot{L}_{S} / \dot{L}_{T} \leq \rho\left(\dot{L}_{S} / \dot{L}_{T} \geq \rho\right)$, respectively.

Suppose some configuration $Y$ in $\Delta_{0}$ existed for which $\rho$ achieved a minimum at a value $\rho<\sqrt{3} / 2$.

(1) If we can find a vector $v$ at $Y$ for which $\dot{L}_{T}<0$ and $\dot{L}_{S}<0$ but $\dot{L}_{S} / \dot{L}_{T} \geq$ $\sqrt{3} / 2>\rho$, then $Y$ is not a minimum for $\rho$.

(2) In [1] it is shown that $\rho>0.82416 \ldots$ for Euclidean Steiner minimal trees. If we can find a vector $v$ at $Y$ for which $\dot{L}_{T}>0$ and $\dot{L}_{\mathrm{S}}>0$ but $\dot{L}_{S} / \dot{L}_{T}<$ $0.82<\rho$, then the configuration is not a minimum for the ratio. 
(3) Note that in the case where we can find a vector $v$ at $Y$ such that $\dot{L}_{T} \geq 0$ and $\dot{L}_{S}<0$ we cannot also be at a minimum.

To summarize: if for every $Y$ in $\Delta_{0}$ we can find a variation vector $v$ at $Y$ belonging to one of the above three cases, then we have shown that there cannot be any cocircular configuration with $\rho<\sqrt{3} / 2$.

\section{The Steiner Ratio Conjecture for Cocircular Points}

Theorem. The Steiner ratio conjecture, $\rho \geq \sqrt{3} / 2$, is true for $n$ cocircular points $x_{1}, x_{2}, \ldots, x_{n}$. Also, $\rho=\sqrt{3} / 2$ is achieved only when there are exactly three distinct points lying at the vertices of an equilateral triangle.

Remarks. (1) We leave the discussion of the case $\rho=\sqrt{3} / 2$ to the end of the proof.

(2) From now on, it is supposed that $x_{1}, x_{2}, \ldots, x_{n}$ are cyclically ordered around the unit circle. The indices can always be changed to achieve this. Also, it suffices to assume that the points are all distinct, by a simple induction argument.

(3) In [7] we have established the ratio conjecture for six points. So it is convenient to restrict attention to configurations where $n \geq 7$.

Proof. It can be easily shown that a minimal spanning tree $T$ for $n$ cocircular points $x_{1}, x_{2}, \ldots, x_{n}$ is the union of the edges $x_{i} x_{i+1}, i=1, \ldots, n-1$, and $x_{n} x_{1}$, excluding a longest edge. Let $P$ denote the polygon consisting of all the edges $x_{i} x_{i+1}$ plus $x_{n} x_{1}$ for $i=1, \ldots, n-1$.

An end of the Steiner tree $S$ is a pair of points $x_{j}, x_{j+1}$ or $x_{n}, x_{1}$ which are adjacent to a Steiner vertex of $S$ (see Fig. 1). As we can assume that $S$ is full (see the remarks at the start of Section 1), $S$ has at least two ends. It is at these ends of the Steiner tree that we do our variations. For convenience, indices $n+1$, $n+2, \ldots$ are taken modulo $n$ to denote $1,2, \ldots$

Lemma. If $n \geq 8$, then $S$ has two different ends $x_{j}, x_{j+1}$ and $x_{k}, x_{k+1}$ for which $1<j<k$ and all the points $x_{j-1}, x_{j}, x_{j+1}, x_{j+2}, x_{k}, x_{k+1}$ are distinct.

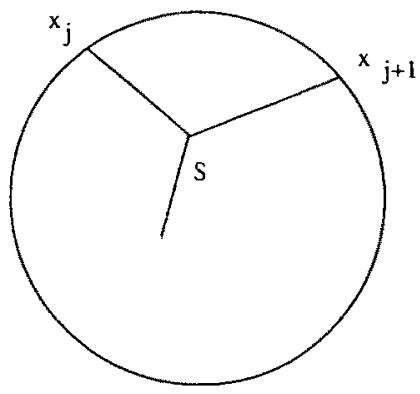

Fig. 1 


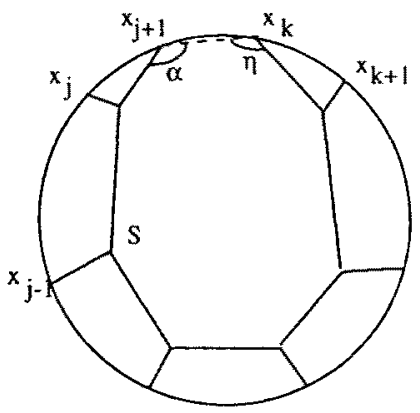

Fig. 2

Proof. Since $S$ is full, two distinct ends $x_{j}, x_{j+1}$ and $x_{k}, x_{k+1}$ can be found so that $1<j<k$, by a suitable choice of indices.

Case 1. If $x_{j+1}=x_{k}$, then it follows that $n=3$.

Case 2. Suppose $x_{j+2}=x_{k}$ and $S$ has no other ends. Then angle considerations either give $n=7$, with the only full topology for $S$ as in Fig. 2, or $n \leq 6$.

Case 3. Assume $x_{j+2}=x_{k}$ and $S$ has a third end $x_{p}, x_{p+1}$ with $j<k<p$, without loss of generality. The result follows using two of the three ends, unless $x_{k+2}=x_{p}$ and $x_{p+2}=x_{j}$, but in this case $n=6$. This completes the proof of the lemma.

Our plan is first to deal with configurations where $n \geq 8$. By the lemma, there are two ends $x_{j}, x_{j+1}$ and $x_{k}, x_{k+1}$ with $j+2<k$. Our variation $v$ is a pair of vectors chosen at $x_{j}$ and $x_{j+1}$ tangent to the circle (see Fig. 3).

(I) Edge $x_{j} x_{j+1}$ has length at most $\sqrt{3}$.

Subcase (a). Neither of the edges adjacent to $x_{j} x_{j+1}$ is longest in the polygon $P$.

We compute $L_{S}$ and $\dot{L}_{T}$. The basic principle, from [6], is that the contribution of an edge to a derivative is minus the cosine of the angle between $v$ and the edge, assuming $v$ has a unit vector component at some end of the edge. Such contribu-

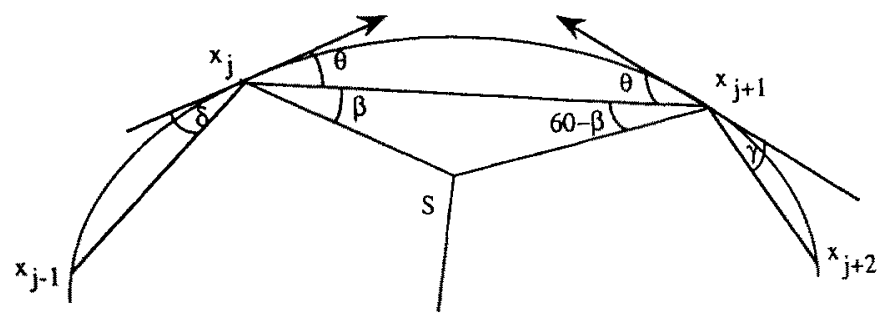

Fig. 3 
tions are then summed. Let $\beta$ and $\theta$ be the angles indicated in Fig. 3. Then $\beta \leq 60^{\circ}$ and

$$
-\dot{L}_{S}=\cos (\theta+\beta)+\cos \left(60^{\circ}-\beta+\theta\right)>\cos \left(60^{\circ}+\theta\right)+\cos \theta
$$

The latter inequality follows since $\theta \leq 60^{\circ}$ by the assumption that length $x_{j} x_{j+1} \leq \sqrt{3}$.

$$
\begin{aligned}
-\dot{L}_{T} & =2 \cos \theta+\cos \left(180^{\circ}-\gamma\right)+\cos \left(180^{\circ}-\delta\right) \\
& =2 \cos \theta-\cos \gamma-\cos \delta,
\end{aligned}
$$

in case $x_{j} x_{j+1}$ is not a unique maximum length edge in $P$. Note that if $x_{j} x_{j+1}$ is a unique longest edge in $P$, then $x_{j} x_{j+1}$ is not in $T$ and so does not contribute to the derivative of length. Consequently, $-\dot{L}_{T}=-\cos \gamma-\cos \delta$ in this latter case.

As $\dot{L}_{S}<0$, if $\dot{L}_{T}>0$, then the configuration is not a minimum for $\rho$, by case (3) in Section 1. This covers the possibility that $x_{j} x_{j+1}$ is the single longest edge in $P$. If $\dot{L}_{T}<0$, we need to show that $-\dot{L}_{S} \geq(\sqrt{3} / 2)\left(-\dot{L}_{T}\right)$.

The desired inequality is

$$
\cos \left(\theta+60^{\circ}\right)+\cos \theta \geq(\sqrt{3} / 2)(2 \cos \theta-\cos \gamma-\cos \delta),
$$

i.e.,

$$
\frac{3}{2} \cos \theta-(\sqrt{3} / 2) \sin \theta \geq \sqrt{3} \cos \theta-(\sqrt{3} / 2) \cos \gamma-(\sqrt{3} / 2) \cos \delta,
$$

i.e.,

$$
(\sqrt{3} / 2)(\cos \gamma+\cos \delta) \geq(\sqrt{3} / 2) \sin \theta+(\sqrt{3}-3 / 2) \cos \theta .
$$

The right-hand side is maximized when $\theta=60^{\circ}$, by $(\sqrt{3} / 2)^{2}+(\sqrt{3}-3 / 2) 1 / 2=$ $\sqrt{3} / 2$. Note that $\theta$ has domain $0^{\circ} \leq \theta \leq 60^{\circ}$, by the supposition that length $x_{j} x_{j+1} \leq \sqrt{3}$. It follows that $\cos \gamma+\cos \delta \geq 1$ suffices to establish (A).

From the graphs of $\cos \gamma+\cos \delta=1$ and $\gamma+\delta=100$ (see Fig. 4) it is easy to see that $0^{\circ} \leq \gamma \leq 88^{\circ}, 0^{\circ} \leq \delta \leq 88^{\circ}$, and $\gamma+\delta \leq 100^{\circ}$ imply $\cos \gamma+\cos \delta \geq 1$.

Suppose $\delta>88^{\circ}$. We know it is less than $90^{\circ}$ as the edge at $\delta$ is not longest in $P$ by assumption. Suppose the angles subtended at the center of the circle by $x_{j-1} x_{j}, x_{j} x_{j+1}$, and $x_{j+1} x_{j+2}$ total less than $200^{\circ}$. (This is all we need to deal with (II) below.) The edges subtend angles at the center of twice the angle to the tangent. Then $\theta<12^{\circ}$ and the right-hand side of $(\mathrm{A})$ is maximized by

$$
(\sqrt{3} / 2) \sin 12^{\circ}+(\sqrt{3}-3 / 2) \cos 12^{\circ}=0.4070 .
$$

We need $(\sqrt{3} / 2)(\cos \gamma+\cos \delta) \geq 0.4070$, i.e.,

$$
\cos \gamma+\cos \delta \geq 0.4700 \text {. }
$$

Since $\gamma<12^{\circ}, \cos \gamma \geq 0.9781>0.4700$ and the inequality holds. 


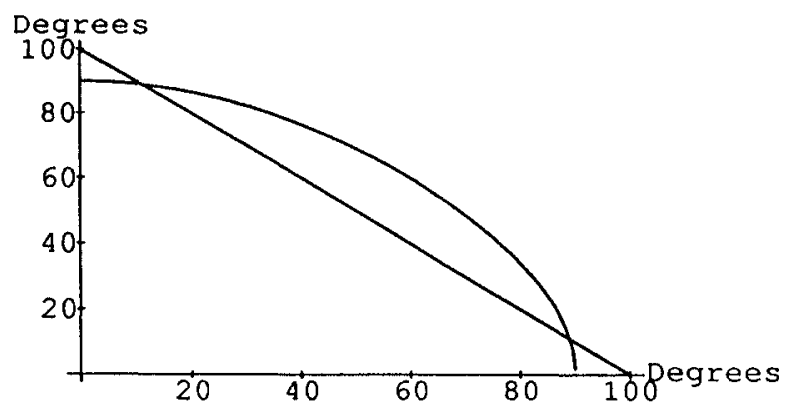

Fig. 4. The graphs of $\cos \gamma+\cos \delta=1$ and $\gamma+\delta=100$.

On the other hand, assume $\gamma+\delta>100^{\circ}$. So together the edges $x_{j-1} x_{j}, x_{j} x_{j+1}$, and $x_{j+1} x_{j+2}$ subtend at least $200^{\circ}$ at the center. If the edge $x_{k} x_{k+1}$ has length at most $\sqrt{3}$, then we can work at the end $x_{k}, x_{k+1}$, since the edges $x_{k-1} x_{k}, x_{k} x_{k+1}$, and $x_{k+1} x_{k+2}$ subtend less than $200^{\circ}$. So this subcase is complete.

Subcase (b). At least one of the edges adjacent to $x_{j} x_{j+1}$ is long in $P$. By this we mean that $P$ may have one longest or several equally long edges and at least one of these is next to $x_{j} x_{j+1}$. Note that in this case, since the edges $x_{j-i} x_{j}$ and $x_{j+1} x_{j+2}$ lengthen during the variation $v$, the longest side will not contribute to $L_{T}$. Consequently, $-\dot{L}_{T}=2 \cos \theta-\cos \gamma$, say, where $\gamma \leq \delta$. From (A), the inequality needed is

$$
(\sqrt{3} / 2) \cos \gamma \geq(\sqrt{3} / 2) \sin \theta+(\sqrt{3}-3 / 2) \cos \theta
$$

If the three edges $x_{j-1} x_{j}, x_{j} x_{j+1}$, and $x_{j+1} x_{j+2}$ subtend less than $200^{\circ}$ at the center of the circle, then $\theta+\gamma+\delta<100^{\circ}$. Now $\gamma \leq \delta$. Also reducing $\gamma$ certainly increases $\cos \gamma$. So it suffices to prove that if $\theta+2 \gamma=100^{\circ}$, then (B) holds. This is shown graphically in Fig. 5.

From the graph, we know the inequality (B) is valid if $\theta \leq 40^{\circ}$, where $\theta+2 \gamma \leq 100^{\circ}$ and the three angles $\theta, \gamma, \delta$ sum to at most $100^{\circ}$.

We need only consider $\theta$ in the range $0^{\circ} \leq \theta \leq 50^{\circ}$, since if $\theta>50^{\circ}$, then $\theta+\delta>100^{\circ}$, since $2 \delta$ is subtended by the longest edge in the polygon $P$.

Now if $40^{\circ} \leq \theta \leq 50^{\circ}$, then $\delta \geq 40^{\circ}$ and $\theta+\gamma+\delta<100^{\circ}$ gives $\gamma<20^{\circ}$. Consequently, in (B),

$$
\begin{aligned}
& \text { LHS }>(\sqrt{3} / 2) \cos 20^{\circ}=0.8138 \\
& \text { RHS }<(\sqrt{3} / 2) \sin 50^{\circ}+\left(\sqrt{3}-\frac{3}{2}\right) \cos 50^{\circ}=0.8126 .
\end{aligned}
$$

Thus the inequality holds.

Putting the two subcases (a) and (b) together, we conclude that if there is an end $x_{j}, x_{j+1}$ so that length $x_{j} x_{j+1} \leq \sqrt{3}$ and the edges $x_{j-1} x_{j}, x_{j} x_{j+1}$, and 


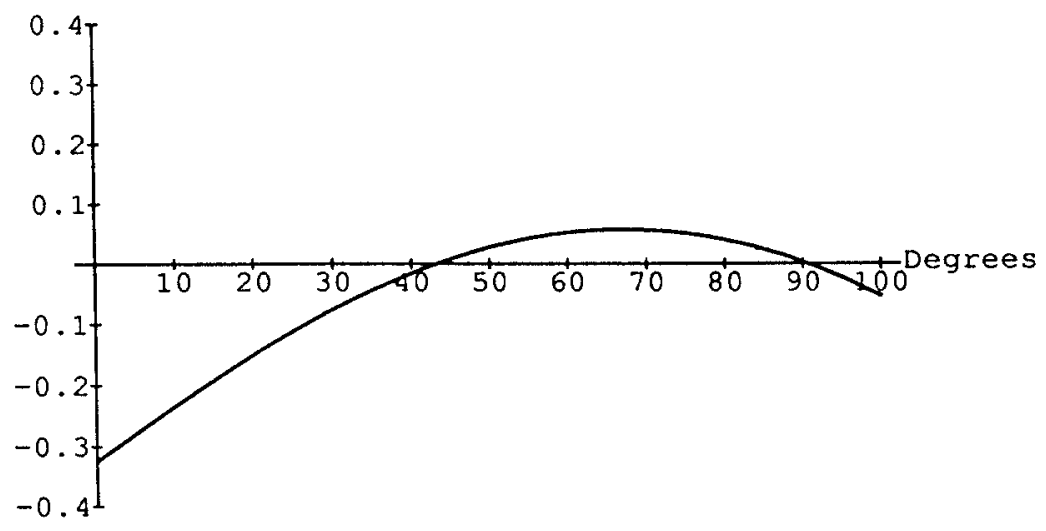

Fig. 5. $f(\theta)=\left(\sqrt{3}-\frac{3}{2}\right) \cos \theta+(\sqrt{3} / 2)(\sin \theta-\cos (50-\theta / 2))$.

$x_{j+1} x_{j+2}$ together subtend at most $200^{\circ}$ at the center of the circle, then the configuration cannot minimize $\rho$ with a value $\leq \sqrt{3} / 2$.

(II) Assume that the edge $x_{j} x_{j+1}$ at an end has length $>\sqrt{3}$. Then $x_{j} x_{j+1}$ subtends at least $120^{\circ}$ at the center of the circle. By the lemma, if $n \geq 8$ we can choose the end $x_{j}, x_{j+1}$ or $x_{k}, x_{k+1}$ where the three adjacent edges subtend a total angle at the center of at most $180^{\circ}$. Note by (I), if this end is say $x_{k}, x_{k+1}$ and length $x_{k} x_{k+1} \leq \sqrt{3}$, then the conclusion is that $\rho$ cannot achieve a minimum of $<\sqrt{3} / 2$ at this set of points. So it is sufficient to suppose that $x_{j-1} x_{j}, x_{j} x_{j+1}$, and $x_{j+1} x_{j+2}$ subtend angles which sum to less than $180^{\circ}$. It follows that the edges $x_{j-1} x_{j}$ and $x_{j+1} x_{j+2}$ cannot be longest in $P$, since they subtend at most $60^{\circ}$ at the center.

We compute $\dot{L}_{T}=\cos \gamma+\cos \delta>0$, if $x_{j} x_{j+1}$ is the unique longest edge in $P$. On the other hand, if $x_{j} x_{j+1}$ is short or equally long, then it contributes to $\dot{L}_{T}$ and we obtain

$$
\dot{L}_{T}=\cos \gamma+\cos \delta-2 \cos \theta>0
$$

Moreover, as $\theta>60^{\circ}, 0^{\circ} \leq \gamma, 0^{\circ} \leq \delta, \gamma+\delta<30^{\circ}$ implies

$$
\begin{aligned}
\dot{L}_{T} & =\cos \gamma+\cos \delta-2 \cos \theta \\
& =2(\cos (\gamma+\delta) / 2)(\cos (\gamma-\delta) / 2)-2 \cos \theta \\
& >2 \cos 15^{\circ} \cos 15^{\circ}-1 \\
& =\sqrt{3} / 2 .
\end{aligned}
$$

Also in the former case,

$$
\dot{L}_{T}=\cos \gamma+\cos \delta>\sqrt{3} / 2+1
$$


by the same argument. On the other hand,

$$
\dot{L}_{\mathrm{S}}=-\cos (\theta+\beta)-\cos \left(60^{\circ}-\beta+\theta\right) \text {. }
$$

The maximum positive value of $\dot{L}_{S}$ occurs when $\theta=90^{\circ}$ and then

$$
\dot{L}_{\mathrm{S}}=\cos \left(90^{\circ}-\beta\right)+\cos \left(\beta+30^{\circ}\right) .
$$

This is maximized when $\beta=30^{\circ}$, i.e.,

$$
\dot{L}_{S}=2 \cos 60^{\circ}=1 \text {. }
$$

Note that $60^{\circ}<\theta \leq 90^{\circ}$ and $0^{\circ}<\beta<60^{\circ}$. However, $\dot{L}_{T}>\sqrt{3} / 2$ and $\dot{L}_{S}=1$ does not give the desired inequality $\dot{L}_{S}<0.82 \dot{L}_{T}$ of case (2) in Section 1 . However, we can reduce the domain of $\theta$ to achieve the required estimate. Now $\dot{L}_{\mathrm{S}}<-2$ $\cos \left(\theta+30^{\circ}\right)$, from (C), as $0^{\circ}<\beta<60^{\circ}$. Also we know that $L_{T}>\sqrt{3} / 2$. If $\theta \leq 80^{\circ}$, then $\dot{L}_{S}<-2 \cos 110^{\circ}=0.6840$. Moreover, $0.82 \dot{L}_{T}>(0.82)(\sqrt{3} / 2)=0.7101$. So the inequality of case (2) in Section 1 is established and $\rho$ cannot have a minimum at this configuration.

If $\theta>80^{\circ}$, then the angle at the center subtended by $x_{j} x_{j+1}$ is greater than $160^{\circ}$. We have less than $200^{\circ}$ in the complement of this angle at the center. We look at the other end $x_{k}, x_{k+1}$ of the Steiner tree.

The preceding argument in (I) applies at such an end, if the length of $x_{k} x_{k+1}$ is at most $\sqrt{3}$. It therefore remains to consider the two ends $x_{j}, x_{j+1}$ and $x_{k}, x_{k+1}$ both having edge length greater than $\sqrt{3}$. As well, by the previous discussion in (II), we know that say $x_{j} x_{j+1}$ must subtend at least $160^{\circ}$ at the center. Therefore, working at the end $x_{k}, x_{k+1}$, it follows that the previous inequalities all hold except that we must allow $\gamma+\delta$ to vary in the range $0^{\circ}<\gamma+\delta<40^{\circ}$, since now $\theta+\gamma+\delta \leq 100^{\circ}$. Redoing the calculations, case (2) in Section 1 is valid unless $\theta>78^{\circ}$. So $x_{k} x_{k+1}$ subtends more than $156^{\circ}$ at the center of the circle.

To complete the discussion, we look at the ratio $L_{S} / L_{T}$ directly using Melzak's algorithm.

From Fig. 6 we see that

$$
L_{S}>\sqrt{3} \sin \theta+\sqrt{3} \sin \theta^{\prime},
$$

where $2 \theta$ (resp. $2 \theta^{\prime}$ ) is the angle subtended by $x_{j} x_{j+1}$ (resp. $x_{k} x_{k+1}$ ) at the center of the circle. Here the angles lie in the ranges $80^{\circ} \leq \theta \leq 102^{\circ}$ and $78^{\circ} \leq \theta^{\prime} \leq 100^{\circ}$. So we can write $L_{S}>2 \sqrt{3} \sin 78^{\circ}=3.3884$.

On the other hand, it tollows from Fig. 6 that $L_{T}<2 \pi\left(1-\left(\theta+\theta^{\prime}\right) / 180^{\circ}\right)+$ $\sin \theta+\sin \theta^{\prime}$, where $\theta, \theta^{\prime}$ lie in the above ranges. We can roughly estimate an upper bound for $L_{T}$ by

$$
2 \pi\left(1-158^{\circ} / 180^{\circ}\right)+2 \sin 90^{\circ}=2.7679 .
$$




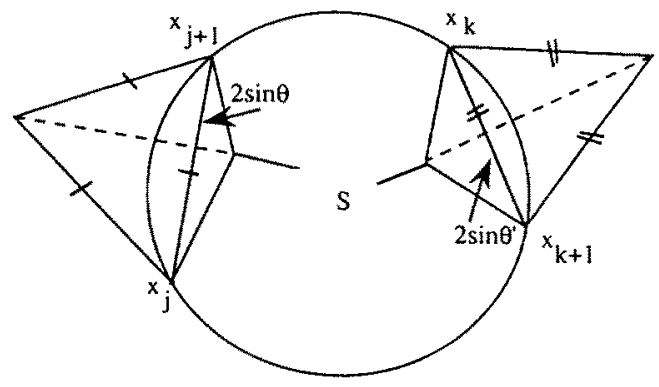

Fig. 6

Therefore $L_{S} / L_{T}>1$. We conclude that there is no minimum configuration for which $\rho<\sqrt{3} / 2$ and $n \geq 8$.

(III) Assume now that $n \leq 7$. If $n \leq 6$, the result follows by [7]. If $n=7$ and the conclusion of the lemma holds, then by (I) and (II) above we obtain the desired result. Finally, we must discuss the unique topology full Steiner tree as in Fig. 2, for which the lemma fails.

Now $\alpha+\eta=300^{\circ}$. Without loss of generality assume that $\alpha \geq 150^{\circ}$. Then $x_{j} x_{j+1}$ and $x_{j+1} x_{k}$ can subtend angles of at most $60^{\circ}$ at the center of the circle. In the terminology of Fig. $3, \theta \leq 30^{\circ}$ and $\gamma \leq 30^{\circ}$. However, it is now possible to apply the argument in (I). In fact since the other five edges of the polygon $P$ subtend at least $300^{\circ}$, neither $x_{j} x_{j+1}$ nor $x_{j+1} x_{k}$ can be a longest edge.

If $x_{j-1} x_{j}$ is not longest in $P$, we are in subcase (a) of (I). We require to establish inequality (A). Since $\theta+\gamma \leq 30^{\circ}$, the left-hand side of (A) can be bounded below by $\sqrt{3} / 2 \cos \left(30^{\circ}-\theta\right)$. Consequently, $4($ LHS - RHS $) \geq(9-4 \sqrt{3}) \cos \theta-\sqrt{3} \sin$ $\theta \geq 4 \sqrt{3}-6$, by substituting $\theta=30^{\circ}$. So (A) is valid and the configuration is not a minimum for $\rho<\sqrt{3} / 2$. If $x_{j-1} x_{j}$ is a longest edge in $P$, by subcase (b) of (I), the inequality (B) must be checked. Exactly the same argument as the one we have just used for inequality (A) applies. So we deduce that $\rho \geq \sqrt{3} / 2$ for cocircular points.

The above technique also applies in the case where $\rho=\sqrt{3} / 2$ and $n \geq 7$. Consequently, there are no configurations of at least seven distinct points on the circle for which $\rho=\sqrt{3} / 2$. The case when there are at most six points is covered by $[7]$.

\section{Acknowledgment}

We would like to thank the referees for a number of helpful comments.

\section{References}

1. F. R. K. Chung and R. L. Graham, A new bound for Euclidean Steiner minimal trees, Ann. N.Y. Acad. Sci. 440 (1985), 328-346. 
2. D. Z. Du, F. K. Hwang, and E. N. Yao, The Steiner ratio conjecture is true for five points, J. Combin. Theory Ser. A 38 (1985), 230-240.

3. D. Z. Du, E. N. Yao, and F. K. Hwang, A short proof of a result of Pollak on Steiner minimal trees, J. Combin. Theory Ser. A 32 (1982), 396-400.

4. E. N. Gilbert and H. O. Pollak, Steiner minimal trees, SIAM J. Appl. Math. 16 (1968), 1-29.

5. H. O. Pollak, Some remarks on the Steiner problem, J. Combin. Theory Ser. A 24(1978), 278-295.

6. J. H. Rubinstein and D. A. Thomas, A variational approach to the Steiner network problem, $A n n$. Oper. Res., to appear.

7. J. H. Rubinstein and D. A. Thomas, The Steiner ratio conjecture for six points, J. Combin. Theory Ser. $A$, to appear.

Received November 20, 1989, and in revised form April 16, 1990, and October 15, 1990.

Note added in proof. We note that $\mathrm{D}$. Z. Du and F. K. Hwang have recently given a complete proof of the Steiner ratio conjecture, using a different global approach. 